\title{
Resistencia a carbapenemes en aislamientos de Pseudomonas aeruginosa: un ejemplo de interacción entre distintos mecanismos
}

\author{
Gisela Santella, ${ }^{1}$ Simona Pollini, ${ }^{2}$ Jean-Denis Docquier, ${ }^{2}$ Marisa Almuzara, ${ }^{3}$ \\ Gabriel Gutkind, ${ }^{1}$ Gian Maria Rossolini ${ }^{2}$ y Marcela Radice ${ }^{1}$
}

Forma de citar Santella G, Pollini S, Docquier J, Almuzara M, Gutkind G, Rossolini GM, et al. Resistencia a carbapenemes en aislamientos de Pseudomonas aeruginosa: un ejemplo de interacción entre distintos mecanismos. Rev Panam Salud Publica. 2011;30(6):545-8.

ABSTRACT Objetivo. Identificar la proteína de membrana externa ausente en los aislamientos resistentes y determinar tanto las causas de su ausencia en la membrana, como la presencia de otros mecanismos de resistencia a carbapenemes en aislamientos clinicos de Pseudomonas aeruginosa.

Métodos. Se estudió un brote de 20 aislamientos de P. aeruginosa previamente caracterizados como productores de la metalobetalactamasa IMP-13. Estos aislamientos presentaron igual expresión de la enzima IMP-13, pero solo cinco de ellos fueron resistentes a carbapenemes. En esos cinco aislamientos resistentes se confirmó la ausencia de una proteína de membrana externa. Se secuenciaron oprD y ampC; se identificaron las proteinas de membrana externa por desorción/ionización láser asistida por matriz/espectometría de masa tiempo de vuelo (MALDI-TOF); se determinó el nivel de expresión de OprD, de AmpC y de los sistemas de eflujo tipo Mex, por reacción en cadena de polimerasa en tiempo real, y por último, se determinó la contribución del déficit de OprD a la resistencia a carbapenemes.

Resultados. La proteína de la membrana externa ausente en el grupo $R$ (resistentes a ambos carbapenemes) fue identificada como OprD-TS, pero no se observaron variaciones en su expresión. El gen oprD presentó mutaciones en los cinco aislamientos resistentes. Se observó la misma producción de la enzima tipo AmpC PDC-5 y del sistema de eflujo Mex AB-OprM entre los aislamientos sensibles y resistentes a carbapenemes. Se analizó cómo la presencia conjunta de IMP-13 y el déficit de OprD contribuyen al aumento de la resistencia.

Conclusiones. Distintos mecanismos contribuyen a la resistencia de aislamientos productores de IMP-13 a carbapenemes. La posibilidad de no detectar estos aislamientos productores de IMP-13 representa un riesgo latente de selección de mutantes con mecanismos de resistencia que se suman para aumentar la resistencia a carbapenemes.

Palabras clave Farmacorresistencia microbiana; Pseudomonas aeruginosa; Argentina.

El tratamiento de las infecciones nosocomiales causadas por cepas de Pseudomonas aeruginosa es actualmente un verdadero desafío terapéutico debido a los

\footnotetext{
Universidad de Buenos Aires, Facultad de Farmacia y Bioquímica, Buenos Aires, Argentina. La correspondencia debe dirigirse a Gabriel Gutkind. Correo electrónico: ggutkind@ffyb.uba.ar

2 Universidad de Siena, Departamento de Biología Molecular, Siena, Italia.

3 Hospital Eva Perón, Buenos Aires, Argentina.
}

múltiples mecanismos de resistencia presentes en este agente patógeno. Además, es frecuente la ocurrencia de brotes nosocomiales de infecciones por Pseudomonas aeruginosa (1). Se entiende por brote nosocomial un aumento repentino del número de infecciones causadas, en este caso, por un microorganismo que presenta un perfil de resistencia emergente, no descrito previamente en el hospital Eva Perón de Buenos Aires, Argentina.
La selección del antibiótico más apropiado para comenzar el tratamiento antibacteriano es fundamental cuando se trata de optimizar los resultados clínicos y lograr tanto la disminución de la morbilidad y mortalidad de los pacientes, como reducir el período de estancia hospitalaria y la diseminación del agente patógeno en el hospital. Sin embargo, esa elección no es sencilla, dada la extraordinaria habilidad de este germen pató- 
geno oportunista de desplegar todos sus mecanismos de resistencia para inactivar una variedad de antimicrobianos, aun después de iniciado el tratamiento antibiótico. Los carbapenemes, son los antibióticos betalactámicos con mayor espectro de acción, y en muchos casos la última opción terapéutica frente a las infecciones por P. aeruginosa. La resistencia adquirida a los carbapenemes resulta de la presencia de distintos mecanismos, que incluyen la inactivación enzimática y la disminución de la concentración del antimicrobiano en el sitio blanco. La importancia relativa de cada mecanismo de resistencia es variable y en muchos casos coexisten más de un mecanismo. La inactivación enzimática está mediada por la sobreexpresión de enzimas de tipo AmpC o por la producción de carbapenemasas, las que pueden ser serinoenzimas o metalobetalactamasas (MBL). La alteración en la concentración en el periplasma puede deberse al déficit en la expresión de proteínas de la membrana externa, OprD, o a la sobreexpresión de sistemas de eflujo de tipo MexAB-OprM y MexXY-OprM, habitualmente expresados en los aislados de $P$. aeruginosa, o MexCD-OprJ y MexEF-OprN, que normalmente no se expresan (2).

En este trabajo se analizó la contribución de distintos mecanismos de resistencia a antibióticos carbapenémicos (imipenem y meropenem) en 20 aislamientos clínicos de $P$. aeruginosa clónicamente relacionados y previamente caracterizados como productores de la metalocarbapenemasa IMP-13 $(3,4)$, pero que muestran diferencias fenotípicas en el grado de resistencia a carbapenemes. Si bien en todos los aislamientos se comprobó previamente igual grado de expresión de metalobetacarbapenemasa, solo 5 de los 20 aislamientos estudiados fueron resistentes a carbapenemes; el resto fue sensible según los puntos de corte del Instituto de Estándares Clínicos y de Laboratorio (CLSI, por su sigla en inglés) (5). Estudios del perfil de las proteínas de membrana externa por electroforesis en gel de poliacrilamida con dodecilsulfato sódico (SDS-PAGE) mostraron la ausencia de una proteína de $46 \mathrm{kDa}$ en los cinco aislamientos resistentes (3). El objetivo principal del presente estudio fue identificar la falta de una proteína de membrana externa y determinar tanto las causas de su ausencia en la membrana, como la presencia de otros mecanismos de resistencia. Un segundo objetivo fue evaluar si la ausencia de la proteína mencionada en la membrana externa de los aislamientos resistentes a carbapenemes era suficiente para conferirles resistencia cuando se expresa la MBL IMP-13.

\section{MÉTODOS}

\section{Aislamientos}

Se realizó un estudio retrospectivo y observacional que incluyó 20 aislamientos de $P$. aeruginosa productores de la MBL IMP-13. Estos aislamientos estaban clónicamente relacionados y fueron recuperados durante diciembre de 2004 y diciembre del 2005 en el hospital Eva Perón de Buenos Aires, Argentina. Diecinueve de los aislamientos estudiados se recuperaron de pacientes hospitalizados, y uno, de un dispositivo médico identificado como posible reservorio del microorganismo, ya que se encontró relación clónica entre los aislamientos por electroforesis en gel de campo pulsado (PFGE) siguiendo el protocolo descrito por Santella y colaboradores (3). De los 20 aislamientos, cinco fueron resistentes a ambos carbapenemes (Grupo R), y el resto presentó susceptibilidad disminuida a ellos (Grupo S) según los puntos de corte establecidos por el CLSI para las pruebas de sensibilidad a los antibióticos (3).

\section{Análisis de los mecanismos que disminuyen la concentración del antibiótico en el sitio activo}

Dado que en el perfil de proteínas de membrana externa resuelto por SDSPAGE se observó la ausencia de una banda proteica de $46 \mathrm{kDa}$ en los aislamientos pertenecientes al Grupo $\mathrm{R}$ al compararlos con el Grupo S, se procedió a identificar dicha proteína. Para ello se purificó la banda proteica presente en los aislamientos del Grupo $S$ a partir de los geles de poliacrilamida y se sometió a espectrometría de masa MALDI-TOF (desorción/ionización láser asistida por matriz/espectometría de masa tiempo de vuelo) (6).

El gen codificante de oprD fue amplificado empleando cebadores específicos y el fragmento obtenido fue secuenciado en forma completa. La expresión del gen codificante de OprD en ambos grupos se determinó por amplificación por reacción en cadena de polimerasa (PCR) en tiempo real con cebadores específicos (7). Se empleó el kit comercial Lightcy- cler ${ }^{\circledR}$ DNA Master SYBR Green I (Roche Applied Science) siguiendo las recomendaciones del fabricante y los controles positivos correspondientes (7). La amplificación, cuantificación y análisis se realizaron por triplicado utilizando el instrumento y programa Lightcycler ${ }^{\circledR}$ (Roche Molecular Biochemicals, version 3.5).

Para determinar la contribución del déficit de OprD a la resistencia a carbapenemes en aislamientos productores de esta metaloenzima, se clonó el gen codificador de la enzima IMP-13 en el vector de expresión de amplio rango de huésped pME6001, y se transformó en $P$. aeruginosa PAO1 ( $P$. aeruginosa PAO1 (pME-IMP-13)) y en $P$. aeruginosa TNP065 deficiente en OprD ( $P$. aeruginosa TNP065DOprD (pME-IMP-13)) (8). Se determinó la concentración inhibitoria mínima de imipenem y meropenem en los clones obtenidos, de acuerdo a las normas del CLSI (5).

Los niveles de expresión de los distintos componentes de los sistemas de eflujo de tipo Mex se determinaron por amplificación por PCR en tiempo real empleando el kit comercial y las condiciones mencionadas en el párrafo anterior. Se amplificaron los genes mexB, mexC, mexE, como marcadores de los sistemas MexAB, MexCD, MexEF, respectivamente.

\section{Análisis de las betalactamasas tipo AmpC}

El gen $a m p C$ fue clonado en el vector pLBII (9) y posteriormente secuenciado. Se determinó la expresión génica por PCR en tiempo real en condiciones basales y en presencia de concentraciones subinhibitorias de imipenem y meropenem.

\section{RESULTADOS}

Por espectrometría de masa se determinó que la proteína $46 \mathrm{kDa}$ presente en los aislamientos del grupo Grupo $\mathrm{S}$ y ausente en los aislamientos del Grupo $\mathrm{R}$ corresponde a OprD-TS, una variante alélica del gen de $\operatorname{opr} D$, previamente descrita en un aislamiento de $P$. aeruginosa productor de VIM-2 (10). La expresión del gen de $\operatorname{opr} D$ fue igual en ambos grupos, lo cual muestra que el déficit de esta proteína en los aislamientos del Grupo R no se debe a una alteración de la regulación de la transcripción. La secuencia del gen codificador de OprD en los aislamientos del Grupo $S$ correspondió a la variante oprD-TS, en concordancia con lo 
observado por espectrometría de masa. En los aislamientos del Grupo R, las secuencias del gen oprD presentaron mutaciones en la secuencia de sus nucleótidos. En dos de cinco aislamientos se detectó una deleción puntual que convierte el producto génico en una proteína de finalización prematura, mientras que en tres de cinco aislamientos se observó una deleción de 27 pb, que si bien no afecta el marco de lectura, probablemente afecte la conformación funcional de la proteína debido a la localización de esta deleción sobre el extremo carboxilo terminal.

No se observaron diferencias en la expresión de los distintos sistemas de eflujo de tipo Mex en los aislamientos del Grupo $\mathrm{R}$ en comparación con el Grupo S. Los niveles de expresión de todas las bombas de eflujo analizadas, para aislamientos tanto del Grupo R como del Grupo S, fueron inferiores al del control hiperproductor de sistemas de eflujo (7), e iguales al de la cepa PAO1 utilizada como control de expresión basal, excepto para MexAB-OprM. En este último se observó un ligero aumento en ambos grupos, R y S (3:1), con respecto a la cepa de referencia.

La concentración inhibitoria mínima (CIM) de imipenem y meropenem de los clones $P$. aeruginosa PAO1 (pME-IMP-13) y $P$. aeruginosa TNP065 $\Delta$ oprD (pMEIMP-13), correspondientes a la transformación del vector pME-IMP13 en PAO1 y en la mutante TNP065 deficiente en OprD (TNP065) respectivamente, se muestran en el cuadro 1.

Respecto a la caracterización de la enzima de tipo AmpC, en los aislamientos de ambos grupos se observó que el gen codificador corresponde al de la enzima PDC-5, una variante de AmpC, con actividad débil sobre los carbapenemes (11). En condiciones basales, no se observaron diferencias en la expresión de dicho gen

CUADRO 1. Valores de la concentración inhibitoria mínima de carbapenemes en aislamientos de Pseudomonas aeruginosa PAO1 y P. aeruginosa TNP065 y los respectivos transformados productores de IMP-13

\begin{tabular}{lcccc}
\hline & \multicolumn{4}{c}{$\mathrm{CIM}(\mu \mathrm{g} / \mathrm{ml})$} \\
\cline { 2 - 5 } Carbapenemes & $\begin{array}{c}P . \text { aeruginosa } \\
\text { PAO1 }\end{array}$ & $\begin{array}{c}\text { P. aeruginosa } \\
\text { PAO1 } \\
\text { (pME-IMP-13) }\end{array}$ & $\begin{array}{c}P \text {. aeruginosa } \\
\text { TNP065 } \\
\Delta o p r D\end{array}$ & $\begin{array}{c}P \text {. aeruginosa } \\
\text { TNP065 }\end{array}$ \\
\hline Imipenem & 4 & 16 & 16 & $\Delta$ oprD (pME-IMP-13) \\
Meropenem & 0,5 & 4 & 4 & 32
\end{tabular}

P. aeruginosa PAO1 (pME-IMP-13): P. aeruginosa PAO1 transformada con el plásmido de expresión pME, que contiene el gen codificador de la enzima IMP-13.

$P$. aeruginosa TNP065 $\triangle$ oprD: $P$. aeruginosa TNP065, que contiene una deleción en el gen codificante de la porina oprD. $P$. aeruginosa TNP065 $\Delta$ oprD (pME-IMP-13): $P$. aeruginosa TNP065 $\Delta$ oprD transformada con el plásmido de expresión pME que contienen el gen codificador de la enzima IMP-13.

entre los aislamientos de los Grupos R y $S$. Ante la presencia de antibióticos carbapenémicos como inductores de la expresión de AmpC, se observó un aumento de 9 y 90 veces con imipenem y meropenen, respectivamente, pero sólo en el Grupo S. Este resultado se debe probablemente a que el déficit de OprD en la membrana externa de los aislamientos del Grupo $\mathrm{R}$ restringe el ingreso de los antibióticos inductores, lo cual sumado a la hidrólisis mediada por la enzima IMP-13, resulta en concentraciones que no logran inducir la expresión de AmpC.

\section{DISCUSIÓN}

El análisis conjunto de los resultados del presente trabajo y de las observaciones previas en párrafos anteriores no sólo muestra la coexistencia de distintos mecanismos de resistencia en un mismo aislamiento, sino también la necesidad de que se dé dicha coexpresión para que los microorganismos presenten resistencia fenotípicamente observable. En trabajos anteriores hemos demostrado que la frecuencia de selección de mutantes resistentes a carbapenemes en los aislamientos del Grupo S varía entre $2 \times$ $10^{-8}$ a $1,6 \times 10^{-7}$ para cada uno de los antibióticos carbapenémicos (3), mientras que no se logra obtener mutantes resistentes de $P$. aeruginosa ATCC 9027 ni de aislamientos clínicos no productores de metalobetalactamasa. Estos resultados muestran el riesgo de selección de un segundo mecanismo de resistencia en presencia de un mecanismo preexistente, y la consecuente posibilidad de selección de microorganismos resistentes que podría llevar a falla terapéutica. En este sentido, la adquisición y expresión de la MBL IMP-13 no confiere resistencia a carbapenemes según los puntos de corte del CLSI, pero determina un aumento 
vorecer tanto su diseminación (4), como la administración inadecuada de un tratamiento basado en la interpretación de un antibiograma con los puntos de corte actuales propuestos por el CLSI.

En nuestro país, la Subcomisión de Antimicrobianos de la Sociedad Argentina de Bacteriología, Micología y Parasitología Clínica (SADEBAC), a su vez, parte de la Asociación Argentina de Microbiología (http:/ / www.aam.org.ar/ sadebac.shtml), recomienda analizar la presencia de mecanismos de resistencia en aislamientos de bacilos gramnegativos no fermentadores que presenten puntos de corte de imipenem o meropenem o ambos antibióticos $\leq 21 \mathrm{~mm}$, e incluir en el antibiograma inicial de rutina un disco con ácido etilendiaminotetraacético o EDTA $(1 \mu \mathrm{g})$ a una distancia de $15 \mathrm{~mm}$ del borde de los discos con imipenem y meropenem. Esta fue una herramienta fundamental para detectar y caracterizar los microorganismos estudiados, ya que
15 de los 20 aislamientos correspondían a la categoría sensible según los puntos de corte de difusión en medio sólido del CLSI. No obstante el agrandamiento del halo de inhibición en presencia del quelante puso en evidencia la presencia de la metaloenzima en todos los casos.

Agradecimientos. Agradecemos a Luca Bini, por el servicio brindado en la identificación por MALDI-TOF de la banda proteica.

\section{REFERENCIAS}

1. Rossolini GM, Luzzaro F, Migliavacca R, Mugnaioli C, Pini B, De Luca F, et al. First countrywide survey of acquired metallobeta-lactamases in gram-negative pathogens in Italy. Antimicrob Agents Chemother. 2008;52(11):4023-9.

2. Lister PD, Wolter DJ, Hanson ND. Antibacterial-resistant Pseudomonas aeruginosa: clinical impact and complex regulation of chromosomally encoded resistance mechanisms. Clin Microbiol Rev. 2009;22(4):582-610.

3. Santella G, Cuirolo A, Almuzara M, Palombarani S, Sly G, Radice M, et al. Full resistance and decreased susceptibility to carbapenems in IMP-13-producing Pseudomonas aeruginosa isolates from an outbreak. Antimicrob Agents Chemother. 2010;54(3):1381-2.

4. Santella G, Pollini S, Docquier JD, Mereuta AI, Gutkind G, Rossolini GM, et al. Intercontinental dissemination of IMP-13-producing Pseudomonas aeruginosa belonging in sequence type 621. J Clin Microbiol. 2010;48(11):4342-3.
5. CLSI. Performance Standards for Antimicrobial Susceptibility Testing; Twenty-First Informational Supplement. CLSI document M100-S21. Clinical and Laboratory Standards Institute, 940 West Valley Road, Suite 1400, Wayne, Pennsylvania, 19087 USA, 2011. 2011.

6. Bini L, Pacini S, Liberatori S, Valensin S, Pellegrini M, Raggiaschi R, et al. Extensive temporally regulated reorganization of the lipid raft proteome following T-cell antigen receptor triggering. Biochem J. 2003;369(Pt 2):301-9.

7. Dumas JL, van Delden C, Perron K, Kohler T. Analysis of antibiotic resistance gene expression in Pseudomonas aeruginosa by quantitative real-time-PCR. FEMS Microbiol Lett. 2006;254(2):217-25.

8. Yoneyama H, Yamano Y, Nakae T. Role of porins in the antibiotic susceptibility of Pseudomonas aeruginosa: construction of mutants with deletions in the multiple porin genes. Biochem Biophys Res Commun. 1995;213(1):88-95.
9. Borgianni Luisa FJM, Rossolini GM, Docquier JD, editor. Mutational analysis of the VIM-2 active site: role of position 64 and 87 in enzyme activity and stability. The 46th Conference on Antimicrobial agents and Chemotherapy; 2006; San Francisco, California.

10. Edalucci E, Spinelli R, Dolzani L, Riccio ML, Dubois V, Tonin EA, et al. Acquisition of different carbapenem resistance mechanisms by an epidemic clonal lineage of Pseudomonas aeruginosa. Clin Microbiol Infect. 2008;14(1): 88-90.

11. Rodriguez-Martinez JM, Poirel L, Nordmann P. Extended-spectrum cephalosporinases in Pseudomonas aeruginosa. Antimicrob Agents Chemother. 2009;53(5):1766-71.

Manuscrito recibido el 10 de abril de 2011. Aceptado para publicación, tras revisión, el 14 de noviembre de 2011.

ABSTRACT Objective. To identify the outer membrane protein absent in the resistant isolates and to determine both the causes of its absence in the membrane and the presence of other mechanisms of carbapenem resistance in clinical isolates of Pseudomonas aeruginosa.

Carbapenem resistance in Pseudomonas aeruginosa isolates: an example of interaction between different mechanisms

Methods. Twenty isolates from an outbreak of $P$. aeruginosa previously characterized as metallo-beta-lactamase IMP-13 producers were studied. All the isolates exhibited equal expression of the IMP-13 enzyme, but only five of them were carbapenemresistant. It was found that the five resistant isolates lacked a outer membrane protein. The oprD and $a m p C$ genes were sequenced; the outer membrane proteins were identified using matrix-assisted laser desorption/ionization time-of-flight (MALDI-TOF) mass spectrometry; the OprD and AmpC expressions, as well as the Mex efflux system, were assessed by real-time polymerase chain reaction; and finally, the contribution of reduced OprD to carbapenem resistance was determined.

Results. The absent outer membrane protein in group $\mathrm{R}$ was identified as OprD-TS; however, no variations in its expression were observed. The oprD gene presented mutations in the five resistant isolates. The production of AmpC PDC-5-type enzyme and the MexAB-OprM efflux system was the same in both carbapenem-sensitive and -resistant isolates. The contribution of the combined presence of IMP-13 and reduced OprD to increased resistance was examined.

Conclusions. Different mechanisms contribute to carbapenem resistance in IMP13-producing isolates. The possibility that these IMP-13-producing isolates could go undetected poses a latent risk when selecting mutants with added resistance mechanisms in order to enhance carbapenem resistance.

Key words Drug resistance, microbial; Pseudomonas aeruginosa; Argentina. 